\title{
Cirugía torácica videoasistida uniportal en dos centros universitarios. Experiencia inicial
}

\author{
Valeria Abiuso B. ${ }^{1}$, Jorge Lavanderos F. ${ }^{2}$, Javier Vega S. ${ }^{1}$, Jorge Salguero A. ${ }^{1,2}$, \\ Jaime Fernández R. ${ }^{1}$, Ramón Cardemil H. ${ }^{1}$ y José Miguel Clavero R. ${ }^{1,2}$
}

\section{Initial experience in uniportal video-assisted thoracoscopic surgery at two university campuses}

Objective: To describe the initial results with uniportal Video-Thoracoscopic Surgery (VATS) performed in two campuses by members of the Section of Thoracic Surgery of the University of Chile ("Clínica Las Condes" and University of Chile Clinical Hospital). Materials and Method: Between January 2016 and January 2019, a total of 105 patients underwent uniportal VATS. Clinical data was collected retrospectively from digital records including demographic (age, sex) and clinical variables (diagnosis, surgery, duration of the chest tube, length of stay, conversion rate and postoperative complications). Results: Uniportal VATS was performed on 105 patients during the study period. Twenty-eight cases $(26.6 \%)$ corresponded to lobectomy or anatomic segmentectomy. In 4 cases a 2 nd port was required, 1 patient had to be converted to mini-thoracotomy and 1 to thoracotomy (5.8\% global conversion). Overall, the median length of stay was $3.07 \pm 3.1$ days and the median duration of chest tube drainage was $2.67 \pm 1.61$ days. Seven patients $(6.6 \%)$ presented complications. One patient died due to progression of his disease, there were no deaths related to the procedures. Discussion: Uniportal VATS has similar indications than multiportal VATS. On experienced hands, uniportal VATS has a low morbidity rate. Uniportal VATS appears to produce less post-operative pain, with shorter hospital stay and a faster return to normal life compared to standard VATS. Conclusion: We present the first uniportal VATS series in Chile. Results were similar to published series. Implementation and development of uniportal VATS requires a learning curve similar to any new surgical procedure.

Key words: thoracic surgery; videothoracoscopy; uniportal VATS.

\section{Resumen}

Introducción: Uno de los mayores avances de las últimas décadas en la cirugía de tórax ha sido el desarrollo de la cirugía mínimamente invasiva. Objetivos: Describir la experiencia en videotoracoscopía (VATS) uniportal de miembros del equipo de Cirugía de Tórax de la Universidad de Chile en 2 campos clínicos, (Clínica Las Condes y Hospital Clínico de la Universidad de Chile). Materiales y Método: Se estudiaron 105 pacientes sometidos a VATS uniportal entre enero de 2016 y enero de 2019. Los datos se analizaron de manera retrospectiva considerando variables demográficas (edad, sexo) y clínicas (diagnóstico, cirugía, estadía hospitalaria, días de pleurostomía, conversión y complicaciones). Resultados: De las 105 cirugías realizadas, 28 (26,6\%) correspondieron a cirugías mayores complejas lobectomías y segmentectomías anatómicas. En 4 pacientes se agregó un $2^{\circ}$ puerto, uno se convirtió a minitoracotomía y uno a toracotomía $(5,7 \%$ conversión global). La estadía hospitalaria fue en promedio 3,07 $\pm 3,1$ días y el promedio de mantención de pleurostomía de 2,67 $\pm 1,61$ días. Siete pacientes $(6,6 \%)$ presentaron complicaciones postoperatorias. Un paciente falleció por progresión de su enfermedad, no hubo mortalidad relacionada a la cirugía. Discusión: Las contraindicaciones de la VATS uniportal son las mismas que en la VATS multipuerto. En manos experimentadas, las complicaciones en cirugía por puerto único son bajas. Impresiona tener menos dolor postoperatorio, menor estadía hospitalaria y reintegración precoz a las actividades diarias comparado con la VATS tradicional. Conclusiones: Se presenta la primera serie de VATS uniportal publicada en Chile. Los resultados obtenidos son comparables a los observados en la literatura. Su implementación y desarrollo requiere de una curva de aprendizaje similar a cualquier nueva técnica quirúrgica.

Palabras clave: cirugía torácica; videotoracoscopía; cirugía uniportal.
'Departamento de Cirugía

Hospital Clínico de la Universidad de Chile. Santiago, Chile.

${ }^{2}$ Clínica Las Condes. Facultad de Medicina de la Universidad de Chile. Santiago, Chile.

Recibido el 20 de abril de 2019 y aceptado para publicación el 7 de junio de 2019

Correspondencia a: Dr. José Miguel Clavero drclavero@gmail.com 


\section{Introducción}

Al igual que en otras especialidades quirúrgicas uno de los mayores avances de las últimas décadas en la cirugía de tórax ha sido el desarrollo de la cirugía mínimamente invasiva. Poco después de la introducción de la laparoscopía, se produjo un gran auge en la década de los 90 de la cirugía torácica videoasistida (VATS). Rápidamente se comenzaron a realizar procedimientos cada vez más complejos por esta vía y dadas las ventajas propias de la técnica, la VATS fue reemplazando progresivamente a la toracotomía en la mayoría de los procedimientos quirúrgicos ${ }^{1,2}$.

La primera lobectomía por VATS fue realizada por Roviaro ${ }^{3}$ hace más de 25 años, sin embargo, el desarrollo de las resecciones pulmonares mayores por cirugía mínimamente invasiva fue lento; sólo en la última década se refinaron y estandarizaron las diferentes técnicas de lobectomías y segmentectomías por VATS, extendiéndose progresivamente su uso ${ }^{4-6}$ (Figura 1).

Al ser adoptadas por diferentes centros a nivel mundial, se pudo demostrar que sus resultados oncológicos eran absolutamente comparables a los de la cirugía abierta, pero con las ventajas esperables de los abordajes mínimamente invasivos: menor estadía hospitalaria, menor tasa de complicaciones, reincorporación laboral precoz, mejores resultados en pacientes de edad avanzada y mejor tolerancia a la quimioterapia adyuvante ${ }^{2,6,7}$.

A medida que la técnica mínimamente invasiva se fue adaptando y perfeccionando, varios autores comenzaron a disminuir el número de puertos y limitar el tamaño de la incisión de utilidad, de manera de aumentar aún más los beneficios de la VATS.

Existen múltiples reportes de cirugía por puerto único, siendo Yamamoto et al. el año 1998, uno de los primeros en publicar una serie de 6 pacientes intervenidos por VATS con incisión única de $2 \mathrm{~cm}^{8}$. Luego Migliore et al. el año 2000, presentó una serie de 58 pacientes durante el Simposio Interdisciplinario y de Cirugía Torácica llevado a cabo en Nápoles, Italia ${ }^{9}$.

Es, sin embargo, Gaetano Rocco el año 2000, quien realiza la primera VATS utilizando un puerto único según la técnica conocida actualmente, presentando algunos años después su primera serie de VATS uniportal en diferentes procedimientos diagnósticos y terapéuticos, incluyendo las primeras resecciones pulmonares no anatómicas y tratamiento del neumotórax espontáneo $0^{10-12}$.

En resecciones mayores D'Amico desarrolló la técnica de 2 puertos en lobectomías por VATS, utilizando una incisión anterior de $4-5 \mathrm{~cm}$ en el $5^{\circ}$ espacio intercostal y un puerto posterior en el $7-8^{\circ}$ espacio intercostal ${ }^{13}$. Luego modificó el abordaje manteniendo la incisión anterior, pero cambiando el puerto accesorio a uno anterior, de $5 \mathrm{~mm}$ para la cámara, en el mismo $5^{\circ}$ espacio ${ }^{14}$.

Finalmente, González-Rivas, eliminó el puerto accesorio para la cámara, realizando la primera lobectomía por puerto único el año 2010. Progresivamente fue perfeccionando y extendiendo la técnica a cirugías cada vez más complejas, utilizándola en forma rutinaria para la totalidad de las intervenciones en cirugía torácica, con resultados absolutamente comparables a la VATS convencional y difundiendo la técnica a nivel mundial ${ }^{15-18}$.

En la cirugía uniportal la cámara y todos los
Figura 1. Técnica de VATS clásica. A. Disposición de puertos en diferentes ángulos y visión interna. B. Segmentectomía izquierda. A derecha se introducen 2 pinzas a través de incisión de utilidad, a izquierda el retractor. A6: Arteria segmento superior lóbulo inferior; A2: Arteria segmento posterior lóbulo Superior; AP: Arteria pulmonar.

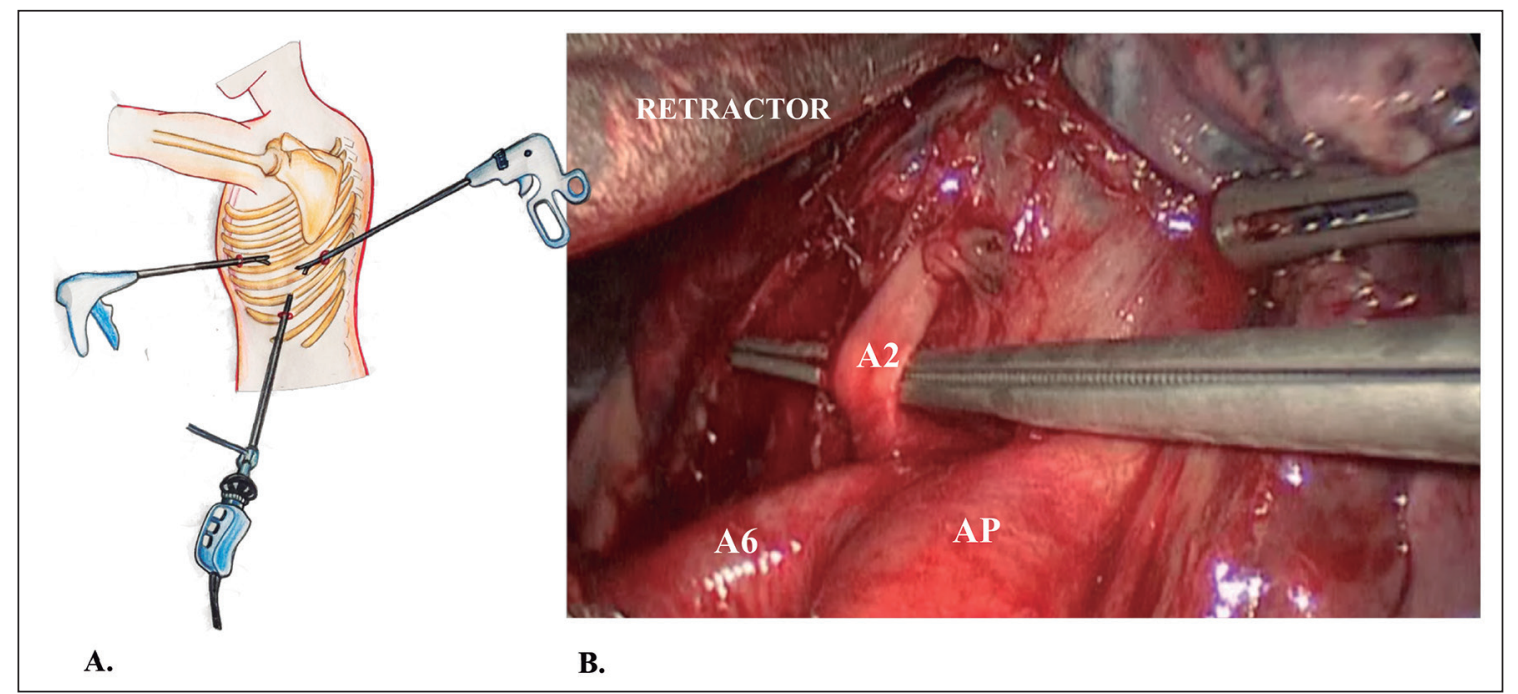


instrumentos se introducen por una sola incisión de $2-4 \mathrm{~cm}$ en el $4^{\circ}$ a $6^{\circ}$ espacio intercostal, anterior al Latissimus Dorsi. La visión que se obtiene es únicamente en el plano sagital, sin plano horizontal ni triangulación como en la VATS convencional. Esto es, sin embargo, una de las ventajas de la técnica, ya que la visualización del objetivo en el mismo plano sagital, que corresponde a la dirección en que se introducen los instrumentos, logra una mejor perspectiva, similar a la visión del cirujano en la cirugía abierta $^{11}$.

Una crítica inicial a la VATS uniportal fue que era difícil de reproducir y con una larga curva de aprendizaje. Rápidamente, sin embargo, al irse difundiendo la técnica, ésta se fue también perfeccionando y estandarizando. Desde entonces, se han realizado múltiples cursos teórico-prácticos a lo largo del mundo e implementado diferentes programas dedicados a su aprendizaje. La técnica puede, actualmente, ser aprendida y reproducida sin mayores dificultades por cirujanos torácicos con formación en videotoracoscopía.

Los centros que han ido adoptando la VATS uniportal han reportado excelentes resultados, del todo comparables a la VATS tradicional. Si bien la VATS uniportal aún no ha demostrado tener ventajas sobre la VATS “estándar”, estas deberían corresponder a las esperadas al reducir aún más el trauma tisular y el dolor postoperatorio, especialmente al eliminar los puertos posteriores ${ }^{19,20}$.

En Chile, la primera VATS por puerto único, se realizó en Valparaíso el año 2013, por los Drs. González y Ávila ${ }^{21}$ y el primer workshop de cirugía uniportal se llevó a cabo en 2016 por los Drs. González-Rivas y Clavero en Santiago durante el congreso de la ALAT ${ }^{22}$. Lentamente, la cirugía uniportal, ha sido adoptada por algunos grupos en el país, pero aún no se han reportado sus resultados.

El objetivo del presente trabajo es presentar la experiencia inicial en cirugía por puerto único por miembros del Equipo de Cirugía de Tórax de la Universidad de Chile en 2 de sus campos clínicos (Hospital Clínico de la Universidad de Chile y Clínica Las Condes).

\section{Materiales y Método}

Revisión retrospectiva descriptiva de todos los pacientes operados mediante VATS uniportal en ambas instituciones, entre enero de 2016 y enero de 2019.

Se revisaron las fichas electrónicas de los pacientes analizando variables demográficas de edad y sexo y variables clínicas como diagnóstico preoperatorio, tipo de cirugía, estadía hospitalaria, días de pleurostomía, necesidad de conversión, adición de puerto extra y complicaciones postoperatorias.

En el período estudiado se realizaron un total de 105 cirugías torácicas videoasistidas por puerto único. El promedio de edad de los pacientes fue de 56 años (rango 15-81), siendo el 54\% de sexo femenino.

\section{Técnica quirúrgica}

Las cirugías se efectuaron con el paciente en decúbito lateral, con anestesia general y ventilación monopulmonar. Se realizó una incisión de 2 a 4 $\mathrm{cm}$ en el $4^{\circ}$ a $6^{\circ}$ espacio intercostal, anterior al Latissimus Dorsi, en la línea axilar media o anterior. Se introdujo en forma simultánea el toracoscopio y demás instrumentos. Dependiendo de la necesidad de mayor instrumentación, se utilizó opcionalmente un anillo retractor de heridas Alexis ${ }^{\circledR}$. Se emplearon ópticas de 5 o $10 \mathrm{~mm}$ de 30 grados asociando instrumentos de energía videotoracoscópicos y de cirugía abierta. Se infiltró con anestésico local el sitio de incisión al comienzo de la operación y bloqueo intercostal bajo visión toracoscópica al final del procedimiento $^{12,15,16,19}$ (Figuras 2 y 3 ).

La técnica quirúrgica varió dependiendo del tipo de cirugía efectuada; la descripción en detalle de los diferentes procedimientos queda fuera del objetivo del presente estudio.

\section{Resultados}

En la Tabla 1 se resumen variables demográficas y clínicas. Los diagnósticos que motivaron la intervención quirúrgica se detallan en la Tabla 2 y las cirugías realizadas se señalan en la Tabla 3.

De las 105 cirugías realizadas, 28 (26,6\%) correspondieron a cirugías mayores complejas: lobectomías o segmentectomías anatómicas (Tabla 3).

En nuestra serie, hubo conversión en 6 de los 105 pacientes, lo que corresponde a un $5,7 \%$ de conversión global. En 4 pacientes se agregó un $2^{\circ}$ puerto, 1 se convirtió a minitoracotomía y 1 a toracotomía póstero-lateral. Al analizar las resecciones pulmonares mayores por separado, el porcentaje de conversión fue de $14,2 \%(4 / 28)$.

Cinco de las 6 conversiones se efectuaron en forma electiva por lenta progresión de la cirugía: en la primera lobectomía operada por el equipo mediante esta técnica, se agregó un segundo puerto por presencia de cisura incompleta y fibrosis del mediastino con ganglios calcificados. El segundo 


\section{ARTÍCULO ORIGINAL}

Figura 2. Técnica de VATS uniportal. A. Visión externa de la disposición de puertos ingresando a través de incisión única. B. Visión interna. Se evidencia ambas pinzas y óptica alineadas ingresando a través de puerto único.

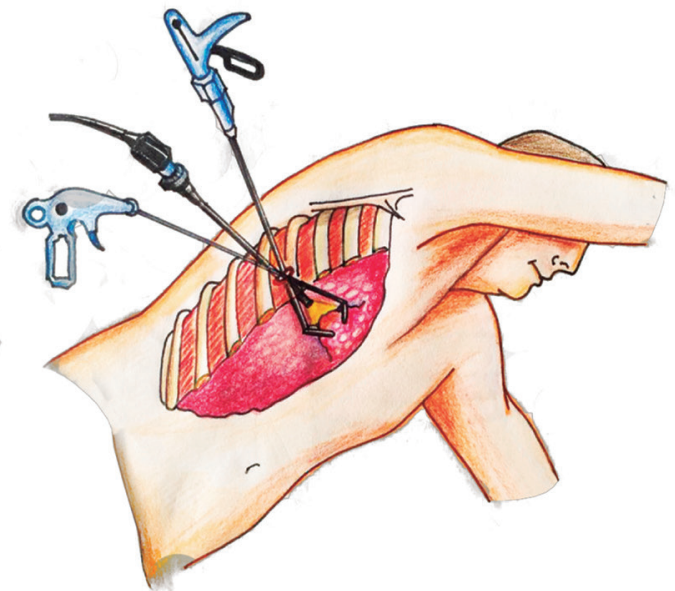

A.



Figura 3. Visión de segmentectomía S9: izquierda (segmento lateral lóbulo inferior). Se evidencia ambas pinzas y óptica alineadas sin triangulación. A8: Arteria segmento basal anterior; A9: Arteria segmento basal lateral; B9: Bronquio segmento basal lateral; A10: Arteria segmento basal posterior; AP: Arteria pulmonar.
Tabla 1. Indicadores demográficos y quirúrgicos

\begin{tabular}{|lcc|}
\hline Variables & $\mathbf{n}$ & $\mathbf{\%}$ \\
\hline Edad & $56,3 \pm 16,9(15-81)$ & \\
Sexo & & \\
$\quad$ Femenino & 57 & 54,28 \\
$\quad$ Masculino & 48 & 45,71 \\
Lateralidad & \\
$\quad$ Derecha & 62 & 59,04 \\
$\quad$ Izquierda & 43 & 40,9 \\
Días de hospitalización & $3,07 \pm 3,1$ & \\
Días de pleurostomía & $2,67 \pm 1,61$ & \\
Complicaciones & 7 & 6,6 \\
\hline
\end{tabular}

Tabla 2. Diagnósticos preoperatorios. Indicaciones quirúrgicas

\begin{tabular}{|lrrl|}
\hline Diagnóstico & n & \% & Especificaciones \\
\hline Nódulos o masas & 40 & 38,1 & $85 \%$ sólidos, 7,5\% mixto, 7,5\% vidrio esmerilado \\
\hline Derrame pleural & 19 & 23,5 & $84,2 \%$ neoplásico, 10,5\% empiema, 5,2\% secundario a TBC \\
\hline Neumotórax & 13 & 12,4 & \\
Metástasis & 16 & 15,2 & Tumor primario: Colon (4 en 3 pacientes), renal (1), vejiga (1) \\
EPD & 9 & 8,5 & \\
\hline Otros & 8 & 7,6 & 3 hemotórax, 1 enf. Crohn, 1 adenopatías mediastínicas, 1 hernia diafragmática, 1 hamartoma, 1 MAV \\
\hline Total & 105 & 100 & \\
\hline
\end{tabular}

EPD: Enfermedad pulmonar difusa; TBC: Tuberculosis; MAV: Malformación arteriovenosa. 
Tabla 3. Tipos de cirugías realizadas

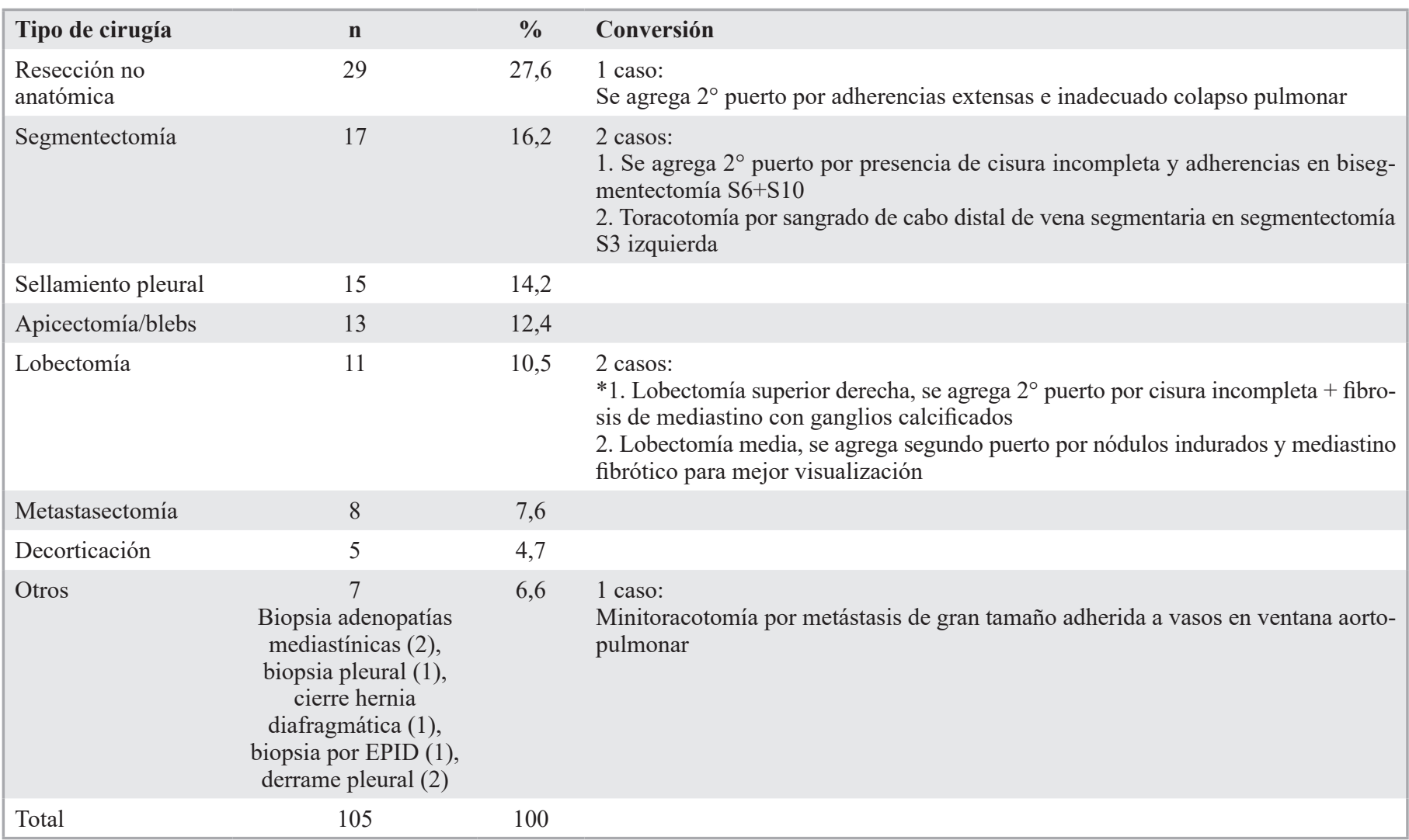

*Primera lobectomía realizada por el equipo.

caso durante bisegmentectomía S6-S10 con cisura incompleta, adherencias extensas e inadecuado colapso pulmonar $\left(2^{\circ}\right.$ puerto se utilizó para cámara e insuflación de $\mathrm{CO} 2$ para colapso pulmonar). En una resección no anatómica por carcinoma in situ, se agregó un segundo puerto por presencia de adherencias múltiples e inadecuado colapso pulmonar. En una lobectomía media se agregó un $2^{\circ}$ puerto por presencia de múltiples adenopatías fibrosas con lenta progresión de la cirugía y finamente se realizó una minitoracotomía en un paciente con metástasis ganglionar de gran tamaño en ventana aortopulmonar con importantes adherencias.

Solamente en un caso se realizó una conversión por sangrado $(0,95 \%)$. En paciente con segmentectomía anatómica S3 izquierda se convirtió a toracotomía por sangrado del cabo distal de vena segmentaria ligada con harmónico, el que no se logró controlar completamente por videotoracoscopía sin comprometer extensión de la resección.

La estadía hospitalaria promedio fue de 3,07 \pm 3,1 días (1-28 días), siendo el promedio de manten- ción de pleurostomía de 2,67 \pm 1,61 días (Tabla 1). Hubo 4 casos puntuales de hospitalización mayor a 7 días; 3 de ellos por causas no relacionadas con el procedimiento quirúrgico torácico y 1 paciente hospitalizado durante 9 días secundario a persistencia de derrame pleural que requirió pleurodesis a través del drenaje pleural.

En relación con la mortalidad, un paciente falleció 13 días después de la cirugía debido a la rápida progresión de su enfermedad de base (cáncer de ovario) con un derrame pleural neoplásico, sin relación directa con el procedimiento quirúrgico.

Se presentaron complicaciones postoperatorias en 7 pacientes: 1 paciente presentó débito prolongado por drenaje postsellamiento, requiriendo una nueva pleurodesis por tubo al $5^{\circ}$ día postoperatorio, retirando el drenaje 4 días más tarde; 1 paciente con reaparición tardía de derrame pleural que requirió drenaje bajo ecografía en forma ambulatoria, 1 paciente presentó paresia de cuerda vocal secundaria postresección de metástasis en ventana aortopulmonar, 1 paciente presentó desaturación transitoria 
de oxígeno al $4^{\circ}$ día postoperatorio que revirtió con manejo médico, 1 paciente tuvo retención urinaria requiriendo cateterismo intermitente, 1 paciente desarrolló un hidroneumotórax secundario a drenaje pleural disfuncional, 1 paciente presentó una arritmia cardiaca por fibrilación auricular que revirtió con manejo médico.

Estas complicaciones corresponden al 6,6\% del total de pacientes analizados en nuestra serie, los cuales según la clasificación de Clavien-Dindo corresponden a 3 pacientes grado I, 2 pacientes grado II, y 2 pacientes grado IIIa23.

\section{Discusión}

Actualmente, la VATS es considerada equivalente a las técnicas abiertas, habiéndose demostrado iguales o mejores resultados oncológicos y mejores resultados en cuanto estadía hospitalaria, complicaciones y reincorporación laboral entre otros ${ }^{6,7}$.

Al igual que lo sucedido con la VATS en relación con las técnicas abiertas, toda nueva técnica debe demostrar tener iguales o mejores resultados que el estándar de tratamiento ${ }^{24}$. La VATS uniportal representa uno de los últimos avances técnicos en cirugía torácica, es producto de modificaciones progresivas de las técnicas de VATS clásica, y ha demostrado rápidamente tener resultados absolutamente comparables a la VATS convencional ${ }^{20,25,26}$.

Las contraindicaciones para el acceso uniportal son las mismas que para la VATS clásica, muchas de las cuales son consideradas actualmente como relativas dependiendo de la experiencia del equipo quirúrgico: adherencias pleurales, cirugía pulmonar previa, antecedente de pleurodesis, radioterapia torácica previa, linfonodos calcificados o de gran tamaño, cisura interlobar fusionada, pulmón enfisematoso, tumores de gran tamaño, adheridos a los vasos o que invaden la pared o estructuras del mediastino e incapacidad del paciente para tolerar la ventilación monopulmonar ${ }^{27}$.

Las causas de conversión electiva, es decir, la continuación de la cirugía por más puertos o cirugía abierta, son en general las mismas que las contraindicaciones relativas. Las causas de conversión de emergencia son generalmente causadas por lesiones vasculares o de la vía aérea, las que muchas veces están subreportadas en la literatura ${ }^{28,29}$.

Diferentes grupos han informado tasas de conversión que van de $2 \%$ a $14 \%$ con la VATS uniportal. En la serie de Rocco de 644 pacientes sometidos a diferentes procedimientos, incluyendo 186 resecciones pulmonares en cuña, 10 requirieron conversión a toracotomía y 14 el uso de trocares adicionales

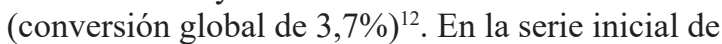
102 resecciones pulmonares mayores publicada por González-Rivas, 3 pacientes requirieron conversión a toracotomía y dos el uso de trocares adicionales (conversión de 4,9\%) ${ }^{16}$. En nuestra serie presentamos una conversión global de 5,7\% y de $14,2 \%$ en resecciones pulmonares mayores.

Al igual que en la VATS clásica, las complicaciones en cirugía uniportal son bajas. Rocco reportó una mortalidad de $0,6 \%$ (todos relacionados a progresión de enfermedad en derrames neoplásicos) y morbilidad de $2,8 \%{ }^{12}$. González-Rivas por su parte, no tuvo mortalidad, presentándose complicaciones en $14 \%$ de los pacientes $(8,65 \%$ correspondió a fuga aérea prolongada), 2 pacientes fueron reoperados por sangrado $^{16}$.

En nuestra serie, un paciente falleció por progresión de un cáncer de ovario posterior a un sellamiento pleural, no hubo mortalidad relacionada directamente a la cirugía. Se presentaron complicaciones en $6,6 \%$ de los pacientes, lo que es comprable con la literatura ${ }^{12,16}$.

En la serie de Rocco la hospitalización media fue de 5,3 días en derrames y de 3,4 días para resecciones en cuña. Los drenajes se retiraron a los 4,3 y 2,4 días respectivamente ${ }^{12}$. En nuestra serie, la estadía hospitalaria fue menor, en promedio 3,2 días para resecciones y/o procedimientos menores y 2,7 días para resecciones mayores (segmentectomías anatómicas y lobectomías). La duración de la pleurostomía fue en promedio 2,7 y 2,6 días respectivamente.

La VATS uniportal, al igual que cualquier nueva técnica quirúrgica, requiere de una curva de aprendi$z \mathrm{zaj}^{30}$. Se recomienda, además de la capacitación directa con cirujanos experimentados, cursos y estudio de la técnica, familiarizarse progresivamente con al abordaje anterior del hilio, retirar posteriormente el puerto posterior, adquiriendo experiencia en VATS biportal y finalmente proceder a la técnica uniportal. Para las lobectomías se recomienda comenzar con lobectomías inferiores, con cisura completa y tumores en estadio inicial ${ }^{31,32}$.

Esta serie representa nuestra experiencia inicial con esta técnica habiendo seguido todos los pasos previamente señalados para su aprendizaje e implementación. Con mayor experiencia hemos logrado ir definiendo mejor las indicaciones y predecir los pacientes de mayor riesgo de complicaciones, en los cuales tenemos un bajo umbral de conversión, lo que no debe considerarse un fracaso de la técnica, sino la cirugía más apropiada y de menos riesgo para casos complejos.

Si bien nuestra serie es de un bajo número de 
pacientes, hemos obtenidos resultados comparables a los presentados en la literatura en cuanto a conversión, complicaciones y estadía hospitalaria ${ }^{2,12,16}$. Es nuestra impresión que VATS uniportal tiene ventajas sobre la VATS clásica en relación al dolor postoperatorio (especialmente por la eliminación del puerto posterior), estadía hospitalaria y reincorporación laboral; lo que deberá ser demostrado en estudios posteriores.

Nuestros resultados muestran que la VATS uniportal puede ser realizada en forma segura en la gran mayoría de los pacientes, logrando una excelente exposición e instrumentación, y permitiendo realizar todo tipo de cirugías complejas al igual que la VATS convencional.

\section{Conclusión}

Este estudio representa la primera serie publicada en Chile de los resultados de la cirugía por videotoracoscopía por técnica uniportal. Los resultados obtenidos son comparables a los descritos en la literatura y con los de la VATS clásica.
Nuestra experiencia muestra que se puede realizar en forma segura en la gran mayoría de los pacientes, logrando una excelente exposición e instrumentación, y permitiendo realizar todo tipo de cirugías complejas al igual que la VATS convencional.

$\mathrm{Su}$ aprendizaje e implementación requiere experiencia en videotoracoscopía y adoptar paso a paso las destrezas necesarias, siendo la curva de aprendizaje similar a cualquier técnica de cirugía mínimamente invasiva.

\section{Responsabilidades éticas}

Protección de personas y animales. Los autores declaran que para esta investigación no se han realizado experimentos en seres humanos ni en animales.

Confidencialidad de los datos. Los autores declaran que en este artículo no aparecen datos de pacientes.

Conflictos de interés: no hay.

\section{Bibliografía}

1. Reinersman JM, Passera E, Rocco G. Overview of uniportal video-assisted thoracic surgery (VATS): past and present. Ann Cardiothorac Surg. 2016;5:112-7.

2. Cattaneo S, Park B, Wilton A, Seshan V, Bains M, Downey R, et al. Use of VideoAssisted Thoracic Surgery for Lobectomy in the Elderly Results in Fewer Complications. The Annals of Thoracic Surgery 2008;85:231-6.

3. Roviaro G, Rebuffat C, Varoli F, Vergani C, Mariani C, Maciocco M. Videoendoscopic pulmonary lobectomy for cancer. Surg Laparosc Endosc. 1992;2:244-7.

4. Mineo T, Ambrogi V. A glance at the history of uniportal video-assisted thoracic surgery. Journal of Visualized Surgery 2017;3:157. Published online 2017 Nov 7. doi: 10.21037/jovs.2017.10.11.

5. Passera E, Rocco G. From full thoracotomy to uniportal videoassisted thoracic surgery: lessons learned. Journal of Visualized Surgery 2017;3:36. Published online 2017 Mar 30. doi: 10.21037/jovs.2017.01.14.
6. Shaw J, Dembitzer F, Wisnivesky J, Litle V, Weiser T, Yun J, et al. Video-Assisted Thoracoscopic Lobectomy: State of the Art and Future Directions. The Annals of Thoracic Surgery 2008;85:S705-9.

7. Scott W, Allen M, Darling G, Meyers B, Decker P, Putman J, et al. Video-assisted thoracic surgery versus open lobectomy for lung cancer: A secondary analysis of data from the American College of Surgeons Oncology Group Z0030 randomized clinical trial. The Journal of Thoracic and Cardiovascular Surgery 2010;139:976-83.

8. Yamamoto H, Okada M, Takada M, Mastuoka H, Sakata K, Kawamura M. Video-Assisted Thoracic Surgery Through a Single Skin Incision. Arch Surg. 1998;133:145-7.

9. Migliore M, Giuliano R, Deodato G. Video assisted thoracic surgery through a single port. Proceedings of Thoracic Surgery and Interdisciplinary Symposium on the Threshold of the Third Millennium, Naples, Italy, May 11-13, 2000; pp 29-30. URL http://xoomer.virgilio.it/naples2000/ index $1 . h$ tml.

10. Rocco G, Martin-Ucar A, Passera E. Uniportal VATS wedge pulmonary resections. The Annals of Thoracic Surgery 2004; 77:726-8

11. Jutley R, Khalil M, Rocco G. Uniportal $v s$ standard three-port VATS technique for spontaneous pneumothorax: comparison of post-operative pain and residual paraesthesia. European Journal of CardioThoracic Surgery 2005;28:43-6.

12. Rocco G, Martucci N, La Manna C, Jones D, De Luca G, La Rocca A, et al. Ten-Year Experience on 644 Patients Undergoing Single-Port (Uniportal) Video-Assisted Thoracoscopic Surgery. The Annals of Thoracic Surgery 2013;96:434-8.

13. Burfeind W, D'Amico T. Thoracoscopic lobectomy. Operative Techniques in Thoracic and Cardiovascular Surgery 2004;9:98-114.

14. Kara H, Balderson S, D'Amico T. Modified uniportal video-assisted thoracoscopic surgery (VATS). Annals of Cardiothoracic Surgery 2016;5:123-6.

15. González D, Paradela M, García J, de la Torre M. Single-port video-assisted thoracoscopic lobectomy. Interactive CardioVascular and Thoracic Surgery 2011;12:514-5.

16. González-Rivas D, Paradela M, Fernández R, Delgado M, Fieira E, Méndez L, et al. 


\section{ARTíCULO ORIGINAL}

Uniportal Video-Assisted Thoracoscopic Lobectomy: Two Years of Experience. The Annals of Thoracic Surgery 2013;95:42632.

17. González-Rivas D, Delgado M, Fieira E, Mández L, Fernández R, de la Torre M. Uniportal video-assisted thoracoscopic pneumonectomy. Journal of Thoracic Disease 2013;5:S246-52.

18. González-Rivas D. Uniportal thoracoscopic surgery: from medical thoracoscopy to non-intubated uniportal video-assisted major pulmonary resections. Annals of Cardiothoracic Surgery 2016;5:85-91.

19. Sihoe A. Uniportal video-assisted thoracic (VATS) lobectomy. Ann Cardiothorac Surg. 2016;5:133-44.

20. Tu C-C, Hsu P-K. Global development and current evidence of uniportal thoracoscopic surgery. Journal of Thoracic Disease 2016;8:11.

21. González C. The adoption of uniportal approach in Chile: the experience of a single surgical team from Valparaíso, Chile. Journal of Visualized Surgery 2016;2:147.

22. Clavero JM. Single port training in LatinAmerica-first uniportal video-assisted thoracoscopic surgery masterclass in Santiago, Chile. Journal of Visualized Surgery 2016;2:140.

23. Seely A, Ivanovic J, Threader J, AlHussaini A, Al-Shehab D, Ramsay T, et al. Systematic Classification of Morbidity and Mortality After Thoracic Surgery. The Annals of Thoracic Surgery 2010;90:93642.

24. Perna V, Carvajal A, Torrecilla J, Gigirey O. Uniportal video-assisted thoracoscopic lobectomy versus other video-assisted thoracoscopic lobectomy techniques: a randomized study. European Journal of Cardio-Thoracic Surgery 2016;50:411-5.

25. Yang Z, Zhai C. Uniportal videoassisted thoracoscopic surgery following neoadjuvant chemotherapy for locallyadvanced lung cancer. Journal of Cardiothoracic Surgery 2018;13, DOI: 10.1186/s13019-018-0714-9.

26. Wang L, Liu D, Lu J, Zhang S, Yang X. The feasibility and advantage of uniportal video-assisted thoracoscopic surgery (VATS) in pulmonary lobectomy. BMC Cancer 2017; 17, DOI: 10.1186/s12885017-3069-z.

27. Sihoe A. Are There Contraindications for Uniportal Video-Assisted Thoracic
Surgery? Thoracic Surgery Clinics 2017;27:373-80.

28. Hanna J, Berry M, D'Amico T. Contraindications of video-assisted thoracoscopic surgical lobectomy and determinants of conversion to open Journal of Thoracic Disease 2013;5:S1829.

29. Flores RM, Ihekweazu U, Dycoco J, Rizk N, Rusch V, Bains M. Video-assisted thoracoscopic surgery (VATS) lobectomy: Catastrophic intraoperative complications. The Journal of Thoracic and Cardiovascular Surgery 2011;142:1412-7.

30. Drevet G, Ugalde Figueroa P. Uniportal video-assisted thoracoscopic surgery: safety, efficacy and learning curve during the first 250 cases in Quebec, Canada. Ann Cardiothorac Surg. 2016;5:100-6.

31. Sandri A, Sihoe A, Salati M, González-Rivas D, Brunelli A. Training in Uniportal Video-Assisted Thoracic Surgery. Thoracic Surgery Clinics 2017;27:417-23

32. Bertolaccini L, Viti A, Terzi A, Rocco G. Geometric and ergonomic characteristics of the uniportal video-assisted thoracoscopic surgery (VATS) approach. Ann Cardiothorac Surg. 2016;5:118-22. 